\title{
The pre-cataclysmic variable, LTT 560
}

\author{
C. Tappert ${ }^{1}$, B. T. Gänsicke ${ }^{2}$, L. Schmidtobreick ${ }^{3}$, A. Aungwerojwit ${ }^{2,4}$, R. E. Mennickent ${ }^{5}$, and D. Koester ${ }^{6}$ \\ 1 Departamento de Astronomía y Astrofísica, Pontificia Universidad Católica, Vicuña Mackenna 4860, $782-0436$ Macul, Chile \\ e-mail: ctappert@astro.puc.cl \\ 2 Department of Physics, University of Warwick, Coventry CV4 7AL, UK \\ e-mail: [Boris.Gaensicke;a.aungwerojwit]@warwick.ac.uk \\ 3 European Southern Observatory, Casilla 19001, Santiago 19, Chile \\ e-mail: 1schmidt@eso.org \\ 4 Department of Physics, Faculty of Science, Naresuan University, Phitsanulok 65000, Thailand \\ 5 Departamento de Física, Universidad de Concepción, Casilla 160-C, Concepción, Chile \\ e-mail: rmennick@astro-udec.cl \\ 6 Institut für Theoretische Physik und Astrophysik, University of Kiel, 24098 Kiel, Germany \\ e-mail: koester@astrophysik.uni-kiel.de
}

Received 16 February 2007 / Accepted 11 August 2007

\section{ABSTRACT}

\begin{abstract}
Aims. System parameters of the object LTT 560 are determined in order to clarify its nature and evolutionary status.
Methods. We apply time-series photometry to reveal orbital modulations of the light curve, time-series spectroscopy to measure radial velocities of features from both the primary and the secondary star, and flux-calibrated spectroscopy to derive temperatures of both components.

Results. We find that LTT 560 is composed of a low temperature $(T \sim 7500 \mathrm{~K})$ DA white dwarf as the primary and an M5.5 \pm 1 mainsequence star as the secondary component. The current orbital period is $P_{\mathrm{orb}}=3.54(07) \mathrm{h}$. We derive a mass ratio $M_{\mathrm{sec}} / M_{\mathrm{wd}}=0.36(03)$ and estimate the distance to $d=25-40$ pc. Long-term variation of the orbital light curve and an additional H $\alpha$ emission component on the white dwarf indicate activity in the system, probably in the form of flaring and/or accretion events.
\end{abstract}

Key words. binaries: close - stars: late-type - white dwarfs - novae, cataclysmic variables

\section{Introduction}

Cataclysmic Variables (CVs) are close interacting binaries with a white-dwarf (WD) primary accreting matter via Roche-lobe overflow from a late-type companion that is on, or at least close to, the main sequence. These systems are thought to form from initially detached binary stars that go through a commonenvelope (CE) phase (Paczyński 1976, and references herein). In the course of its nuclear evolution, the more massive star expands, eventually fills its Roche lobe, and transfers matter onto the late-type dwarf at very high rates of up to $\dot{M}_{1} \sim$ $0.01 \ldots 0.1 M_{\odot} \mathrm{yr}^{-1}$ (e.g., Meyer \& Meyer-Hofmeister 1979; Warner 1995). The CE configuration results, since the secondary star cannot adjust on such short timescales ${ }^{1}$. The CE provides an enhanced braking mechanism, rapidly reducing the binary separation. After the expulsion of the CE, angular-momentum loss due to magnetic braking further reduces the orbital separation between the remaining WD and its red companion (on much longer timescales). Eventually the Roche lobe of the red dwarf shrinks into contact with the stellar surface, thus starting mass transfer and the proper $\mathrm{CV}$ phase. Following the convention of Schreiber \& Gänsicke (2003), a post-CE binary is called a pre$\mathrm{CV}$ if the time between the expelling of the CE and the start of the $\mathrm{CV}$ phase is less than the Hubble time. For a comprehensive overview on CVs and CV evolution see Warner (1995).

1 Note, however, that neither high mass-transfer-rates lead necessarily to a CE, nor a CE phase to a CV (e.g., Beer et al. 2007; de Kool 1992 respectively).
Currently only $\sim 40$ pre-CVs with established orbital periods are known (Morales-Rueda et al. 2005), which is in strong contrast to the over $600 \mathrm{CVs}$ with measured orbital periods that are listed in version 7.7 of the Ritter \& Kolb (2003) catalogue. Furthermore, this small sample is severely biased by observational selection effects towards young systems containing hot white dwarfs and low-mass companions (Schreiber \& Gänsicke 2003).

LTT 560 is listed as a high proper motion system in the Luyten (1957) catalogue. The object was marked as a possible WD by Eggen (1968), and appeared as a nova-like variable in Vogt (1989) and in Downes et al. (1997), the latter using the designation Scl2. Spectroscopy by Hoard \& Wachter (1998) revealed narrow $\mathrm{H} \alpha$ emission and a red continuum that was fitted well by an M5 dwarf. They also found a variable doubling of the $\mathrm{H} \alpha$ emission that led them to suspect a binary nature for LTT 560.

Here we present photometric light curves and time-resolved and flux-calibrated spectroscopy that reveal LTT 560 as being an old and nearby short-period pre-CV containing a very cool white dwarf.

\section{Observations and data reduction}

The photometric data were taken in two runs on 2002-09-22 and on 2002-10-17+18 at the CTIO/SMARTS $0.9 \mathrm{~m}$ telescope using a $2048 \times 2046 \mathrm{CCD}$ and a Kron-Cousins $R$ filter. A scale of $0.396^{\prime \prime} /$ pixel yields a field-of-view of $13.5^{\prime}$. The data 
Table 1. Log of observations. The configuration column for the spectroscopic data states the grism/grating, the wavelength range, and the spectral FWHM resolution. Date and HJD refer to the start of night.

\begin{tabular}{|c|c|c|c|c|c|c|}
\hline Date & HJD & Telescope & Configuration & $n_{\text {data }}$ & $t_{\exp }[\mathrm{s}]$ & $\Delta t[\mathrm{~h}]$ \\
\hline 2002-09-22 & 2452540 & $0.9 \mathrm{~m}$ & $\mathrm{CCD}+R$ & 130 & 120 & 5.67 \\
\hline $2002-10-17$ & 2452565 & $0.9 \mathrm{~m}$ & $\mathrm{CCD}+R$ & 90 & 120 & 3.84 \\
\hline $2002-10-18$ & 2452566 & $0.9 \mathrm{~m}$ & $\mathrm{CCD}+R$ & 90 & 120 & 3.87 \\
\hline 2003-07-19 & 2452840 & NTT & g\#3, 3350-9000 $\mathrm{A}, 8.3 \AA$ & 3 & 400 & 0.25 \\
\hline 2004-11-29 & 2453339 & $4.0 \mathrm{~m}$ & KPGL3, 3500-7300 ̊, $4.2 \AA$ & 27 & 600 & 5.57 \\
\hline
\end{tabular}

were reduced using the quadred package implemented in $\mathrm{IRAF}^{2}$, which takes into account that the CCD is read out via 4 amplifiers. Apart from that, the reduction process is standard, including bias and overscan subtraction, as well as flatfielding. Aperture photometry for all detected stars on the CCD was computed with IRAF's daophot package, and additional daomatch and daomaster (Stetson 1992) routines were used for crossidentification of stellar sources on different CCD frames. The adopted aperture radius was chosen as the one that produced minimum noise in the differential light curve of two comparison stars with brightness similar to the target. An average light curve was computed out of several non-variable comparison stars, and then used to produce differential light curves for all stars in the field (e.g., Howell 1992).

Optical spectroscopy was performed on 2003-07-19 using EMMI on the NTT, at ESO La Silla. Grism \#3 and a 1.0" slit yielded a wavelength range of 3350-9000 $\AA$ and a spectral resolution of $8.3 \AA$. IRAF tasks were used for basic reduction (bias + flats), optimal extraction (Horne 1986), wavelength-, and flux calibration.

Time-resolved spectroscopic data were obtained on 2004-11-29 with the R-C spectrograph mounted on the $4.0 \mathrm{~m}$ Blanco telescope at CTIO. The grating KPGL3 was used with a $1.0^{\prime \prime}$ slit, which yielded a wavelength range of 3500-7300 $\AA$ and a spectral resolution of $4.2 \AA$. Wavelength calibration data were taken with a HeAr lamp every 4 to 5 object spectra. The instrumental wavelength flexure between two calibration exposures was later corrected for by measuring the position of the night sky emission line at $6863 \AA$ and subtracting its variation with respect to the mean. Weather conditions during this run were far from photometric, with a layer of thin clouds during most of the time, so no flux calibration could be performed. Data reduction was carried out as outlined above.

LTT 560 was observed in the near-ultraviolet (NUV) and farultraviolet (FUV) by GALEX as part of its All Sky Survey on October 21, 2003. The $120 \mathrm{~s}$ exposures did not yield a detection in the FUV, but provided an NUV flux of $0.236 \pm 0.01 \mathrm{mJy}$.

A summary of our observations is presented in Table 1.

\section{Results}

\subsection{The flux-calibrated spectrum}

The three individual spectra taken on 2003-07-19 were averaged to yield the spectrum displayed in Fig. 1. It shows a blue continuum with Balmer absorption lines, and a red continuum with molecular absorption bands. The relatively narrow width of the Balmer absorption lines suggests a cold $\left(T_{\text {eff }}<10000 \mathrm{~K}\right)$ white dwarf, and the strength of the TiO bands an intermediate M-type secondary star. On closer inspection, the presence of narrow

\footnotetext{
${ }^{2}$ NOAO PC-IRAF Revision 2.12.2-EXPORT.
}

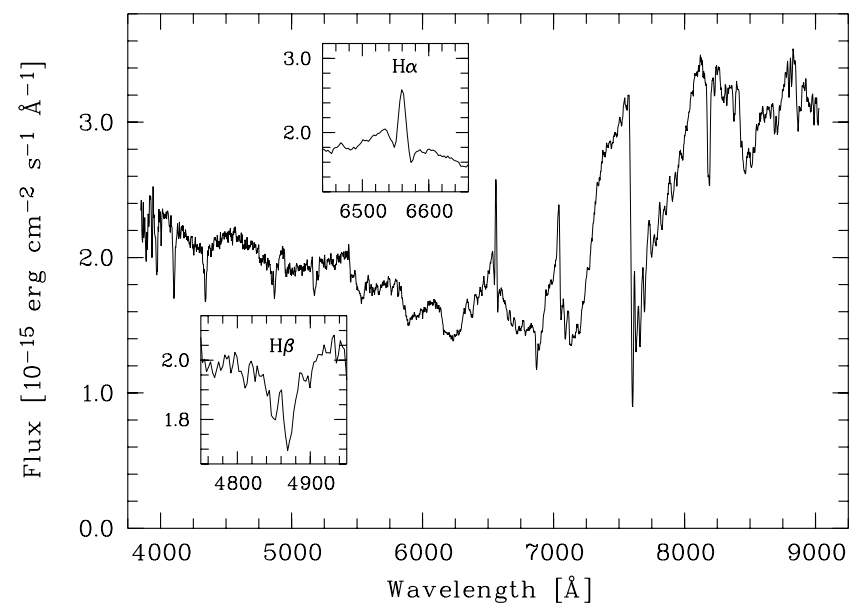

Fig. 1. The average flux-calibrated spectrum. The two insets show close-ups of the regions around $\mathrm{H} \beta$ and $\mathrm{H} \alpha$.

Balmer emission is clearly seen in $\mathrm{H} \alpha$ and $\mathrm{H} \beta$. Convolving the spectrum with Bessell (1990) filter curves yields spectrophotometric magnitudes $V=15.7$ and $R=15.2 \mathrm{mag}$. This is roughly 0.2 mag brighter than previously reported (Hoard \& Wachter 1998, and references therein), but such small difference can probably be attributed to the uncertainties of the flux calibration, and we re-scaled our spectrum of LTT 560 to $V=15.7$ for the subsequent analysis.

We fitted a composite model consisting of a synthetic white dwarf spectrum and an observed M-dwarf template to the fluxcalibrated spectrum of LTT 560 . The synthetic white dwarf spectra were calculated with the code described by Koester et al. (2005) and earlier references given there. We used a mixing length $\mathrm{ML} / 2 \alpha=0.6$, a pure hydrogen composition, a surface gravity $\log g=8.0$, corresponding to a white dwarf mass of $M_{\mathrm{wd}} \simeq 0.55 M_{\odot}$, and covered a temperature range $T_{\mathrm{wd}}=6000 \mathrm{~K}$ to $15000 \mathrm{~K}$. Our library of high signal-to-noise ratio M-dwarf templates covers spectral types M0-M9. These templates were produced by averaging about a dozen high-quality SDSS spectra of each spectral type, where the typing of the SDSS spectra was done based on set of carefully calibrated M-dwarf spectra from Beuermann \& Weichhold (1999). The advantage of our new set of templates over that of Beuermann \& Weichhold (1999) is a homogenous wavelength coverage and spectral resolution for all spectral types. A comparison of our M-dwarf templates to those recently published by Bochanski et al. (2007) showed very good agreement for all spectral types. We scaled the M-dwarf templates to the surface fluxes corresponding to their spectral type using the relation of Beuermann (2006). The best fit to the spectrum of LTT 560 is achieved for a $T_{\mathrm{wd}}=7500 \mathrm{~K}$ white dwarf and either an M5 or M6 secondary star. Figure 2 shows the observed spectrum of LTT 560 along with the white dwarf model, and an M5.5 star obtained from linear interpolation between the 


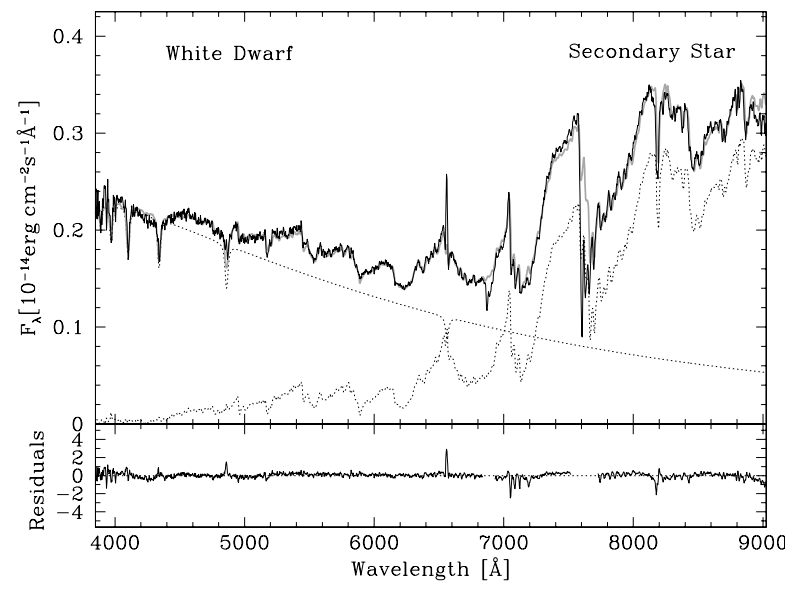

Fig. 2. Two-component fit (thick grey line) to the flux-calibrated spectrum of LTT 560 (solid black line). The individual components, a white dwarf with a temperature of $T_{\mathrm{wd}}=7500 \mathrm{~K}$ and a surface gravity of $\log g=8.0$, and our M5.5 template are shown as dashed lines. The bottom panel shows the residuals of the fit, which highlight the Balmer emission from the M-dwarf.

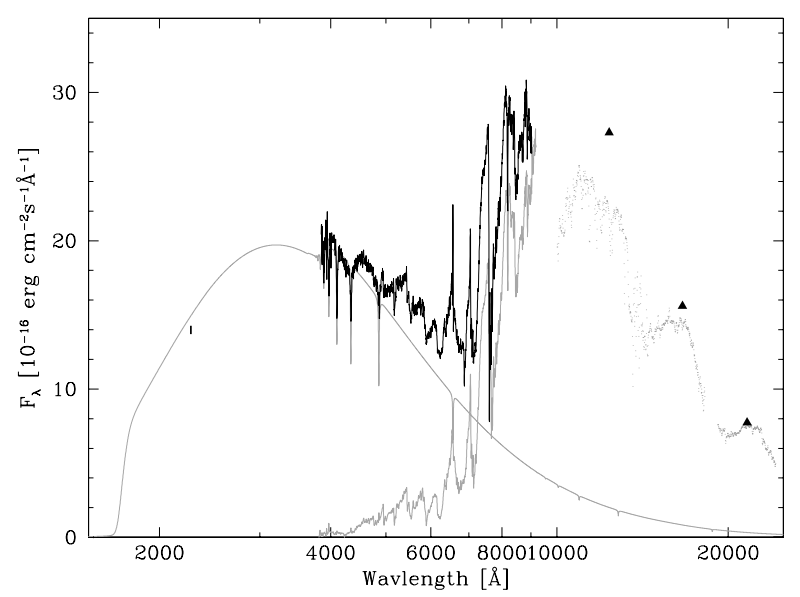

Fig. 3. Spectral energy distribution of LTT 560. The GALEX nearultraviolet flux, the CTIO average spectrum, and the 2MASS $J, H$, and $K_{S}$ fluxes are shown in black. The $T_{\text {wd }}=7500 \mathrm{~K}, \log g=8.0$ white dwarf and the M5.5 M-dwarf from Fig. 2 are plotted as grey lines. The grey dots represent an M5.5 template from S. Leggett's M-dwarf library, scaled according to our fit to the optical data.

M5 and M6 templates, with both components scaled according to the best fit. Earlier and later spectral types for the secondary star result in much poorer fits, and we conclude that the spectral type of the main-sequence companion in LTT 560 is $5.5 \pm 1$. This result is confirmed by recent $K$-band spectroscopy (Tappert et al. 2007).

Our fit to the optical spectrum of LTT 560 agrees well with the 2MASS magnitudes of LTT $560(J=12.65, H=12.13$, and $K_{\mathrm{s}}=11.86$ ) when extending the composite model into the infrared with Leggett's library of M-dwarf $\operatorname{spectra}^{3}$ (Fig. 3). The best-fit white dwarf model spectrum overpredicts the GALEX near-UV flux only by a few per cent. Given the uncertainty in the spectrophotometric calibration of our optical data, and the fact that the 2MASS data have been taken at unknown binary phases, an attempt to fit the overall spectral energy distribution would not result in more accurate stellar parameters, and has therefore not been attempted.

\footnotetext{
${ }^{3}$ http://ftp.jach.hawaii.edu/ukirt/skl/dM. spectra/
}

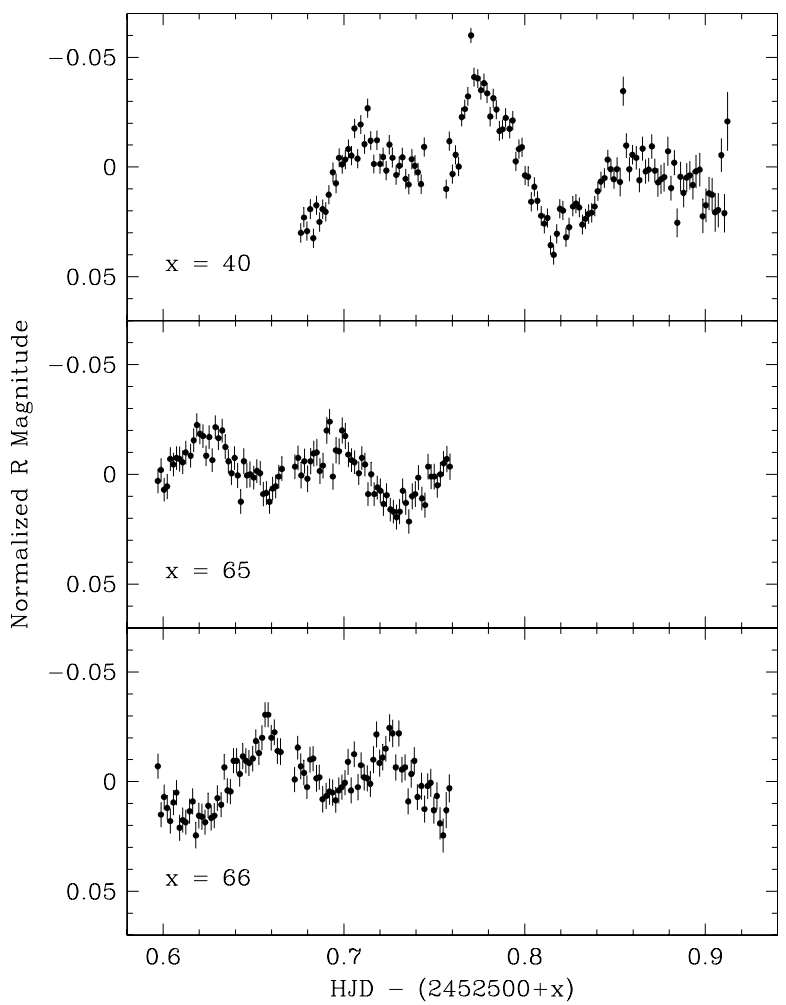

Fig. 4. Individual light curves from 2002-09-22, 2002-10-17, and 200210-18 (top to bottom). The data have been normalised by subtracting the average magnitude of the complete data set.

Assuming a Hamada \& Salpeter (1961) carbon-core massradius relation for the white dwarf, the flux scaling factor between the synthetic spectrum and the data of LTT 560 implies a distance of $d=33 \mathrm{pc}$. This estimate depends somewhat on the white dwarf mass. Repeating the fit for $\log g=7.5\left(M_{\mathrm{wd}}=\right.$ $\left.0.33 M_{\odot}\right)$ and $\log g=8.5\left(M_{\mathrm{wd}}=0.90 M_{\odot}\right)$ results in temperatures lower and larger by $500 \mathrm{~K}$, respectively. The implied distance is $39 \mathrm{pc}$ for $\log g=7.5$ and $26 \mathrm{pc}$ for $\log g=8.5$, and we conclude $25 \mathrm{pc} \lesssim d \lesssim 40 \mathrm{pc}$. This makes the object a good target for a ground-based parallax measurement.

For $d=33 \mathrm{pc}$, the flux scaling factor of our M-dwarf spectral template implies a radius of the secondary of $R_{\mathrm{sec}} \sim$ $8.0 \pm 1.3 \times 10^{9} \mathrm{~cm}$, which is consistent with an $\sim \mathrm{M} 5 \mathrm{~V}$ secondary star (Delfosse et al. 2000; Ségransan et al. 2003). For a mass ratio of $M_{\mathrm{sec}} / M_{\mathrm{wd}}=0.32$ (Sect.3.3) and an orbital period of $3.54 \mathrm{~h}$ (Sect. 3.2), the Roche lobe radius of the secondary star is $\sim 2.1 \times 10^{10} \mathrm{~cm}$, which implies that the secondary star is $\sim 50 \%$ Roche-lobe filling. Given the amplitude of the observed ellipsoidal modulation (Fig. 4), the inferred radius is clearly too small. This will be discussed further in Sect. 4.

\subsection{The light curve}

The resulting light curves of the two observing runs from September and in October 2002 are given in Fig. 4. All three data sets show as a common feature the presence of two minima of different depths, indicating ellipsoidal variation. In the first data set, from September 2002, the two maxima have different values as well.

To explore the periodicity of the light curve, the analysisof-variance (AOV; Schwarzenberg-Czerny 1989) algorithm as implemented in MIDAS was used. The two highest peaks in the periodogram (Fig. 5) correspond to frequencies 


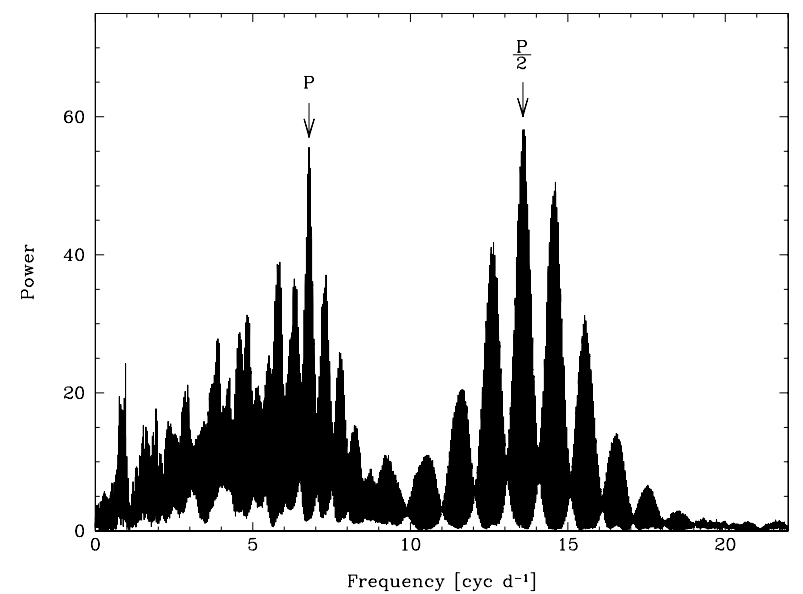

Fig. 5. AOV periodogram of the photometric data. The best period and half its value are indicated.

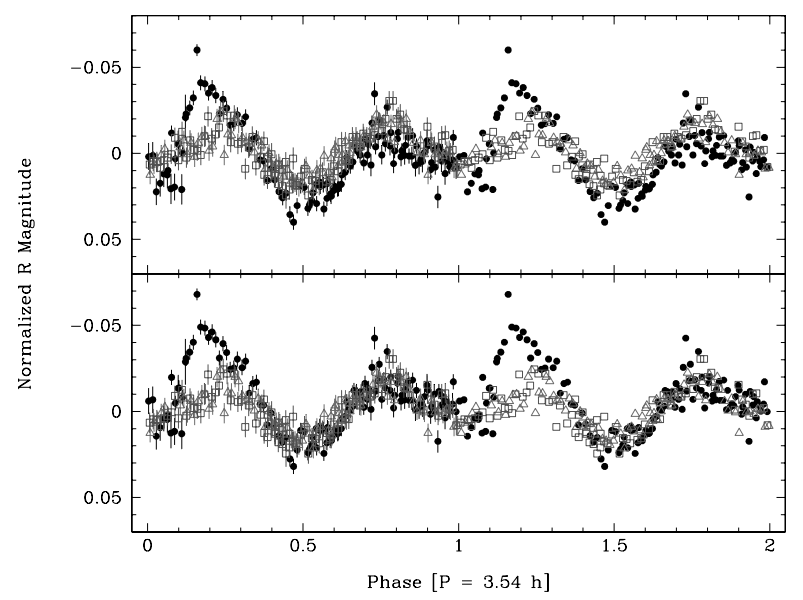

Fig. 6. Light curves folded on the ephemeris in Eq. (1). Filled circles, triangles, and squares, mark data from 2002-09-22, 2002-10-17, and 2002-10-18, respectively. The data are repeated, without error bars, for a second cycle. For the lower plot, an offset of -0.008 mag has been applied to the data from 2002-09-22.

$f_{1}=6.786$ cyc d $^{-1}$ and $f_{2}=13.609$ cyc d $^{-1}$, and thus $f_{2} \approx 2 f_{1}$. We therefore adopt the lower frequency as corresponding to the orbital period, and describe the photometric variation as ellipsoidal: the two minima represent the inferior and superior conjunction of the late-type secondary star, the two maxima are the result of maximum visibility of the Roche-deformed sides of that star at quadrature. From $f_{1}$ we derive a corresponding period $P=3.54(07) \mathrm{h}$, with the uncertainty being estimated from the width of the signal in the periodogram. Figure 6 presents the phase-folded light curve. In principle, there are two effects that can account for the difference in the minima: at superior conjunction either illumination by the white dwarf causes an elevated minimum, or gravitational darkening of the deformed secondary star results in a deeper minimum. Considering the very low temperature of the white dwarf, the latter scenario appears as the most probable one.

Since the orbital phase is now defined we can go on to derive the photometric ephemeris. In the light curve there are three well defined minima that can be fitted with second-order polynomials in order to derive the timings of these minima. These include both the shallow and the deep minimum from 2002-1017 , and the shallow minimum from 2002-10-18. A linear fit to the such derived timings (Table 2) yields the zero point and the
Table 2. Timings of suitable photometric minima.

\begin{tabular}{lll}
\hline \hline Cycle & HJD (2 452 500+) & Error [d] \\
\hline 0.0 & 65.6575 & 0.0097 \\
0.5 & 65.7316 & 0.0055 \\
7.0 & 66.6937 & 0.0064 \\
\hline
\end{tabular}

orbital period. The latter results in $3.55(03) \mathrm{h}$, and thus, within the errors, is identical to the one derived from the periodogram. However, since it is based on only three data points, the formal error of $0.03 \mathrm{~h}$ is probably underestimated. We therefore adopt the period and uncertainties estimated from the periodogram, to obtain the ephemeris

$$
T_{0}(\mathrm{HJD})=2452565.6575(51)+0.1475(29) E,
$$

with $E$ giving the cycle number with respect to the superior conjunction of the white dwarf.

We note that, while the two light curves from October are identical within the photometric errors, the September data set not only presents an enhanced second maximum, but on the whole appears to be displaced to $\sim 0.01 \mathrm{mag}$ fainter magnitudes. Comparison of the average magnitudes of common field stars in the September and the October run yields a standard deviation of $0.008 \mathrm{mag}$, so that this displacement can be explained as being within the photometric precision limit with respect to these two runs. If a corresponding offset of -0.008 mag is applied to the September data, the general shape of that light curve is identical to the October data, with the notable exception of the second maximum (lower plot of Fig. 6). The latter feature is discussed in more detail in Sect. 4.

We used the program Nightfall written by Wichmann ${ }^{4}$ to test the parameters derived from the spectroscopic model (Sect. 3.1) by fitting synthetic light curves to the October 2002 data. A first light curve was calculated adopting the parameters from Table 3, in particular $P_{\text {orb }}=0.1475 \mathrm{~d}, i=60^{\circ}, T_{\mathrm{wd}}=$ $7500 \mathrm{~K}, T_{\mathrm{sec}} \simeq 3000 \mathrm{~K}, M_{\mathrm{wd}} \simeq 0.4-0.6 M_{\odot}, M_{\mathrm{sec}}=0.17 M_{\odot}$, $R_{\mathrm{wd}} \simeq 0.012-0.016 R_{\odot}$, and $R_{\mathrm{sec}}=0.115 R_{\odot}$. However, the ellipsoidal modulation of the resulting light curve is of much lower amplitude $(\sim 0.0015)$ than the observed one. We then fitted the observed light curve by fixing all parameters above but leaving $R_{\text {sec }}$ as a free parameter. We find that, to reproduce an ellipsoidal modulation corresponding to the observed light curve, the required $R_{\mathrm{sec}}$ exceeds the spectroscopic result by a factor of two. The discrepancy between the spectrophotometric radius (Sect. 3.1) and that implied by the ellipsoidal modulation is significant and will be discussed in Sect. 4 .

\subsection{Radial velocities}

The $\mathrm{H} \alpha$ emission line is clearly double-peaked during substantial parts of the orbital cycle (Fig. 7). The trailed spectrogram (Fig. 8) reveals that the two components follow sinusoidal radial velocity variations with a phase difference of $\simeq 0.5$. To measure the radial velocity variation, we fitted the spectra that present two clearly separated $\mathrm{H} \alpha$ components with two Gaussians, finding that these components are unresolved at the spectral resolution of the CTIO spectra.

Next, we attempted to fit all individual spectra with two Gaussians of $F W H M=4.2 \AA$, i.e. with their widths fixed to the

\footnotetext{
4 http://www. lsw. uni-heidelberg.de/ rwichman/ Nightfall.html
} 
Table 3. System parameters of LTT 560.

\begin{tabular}{cccl}
\hline \hline & White dwarf & Main sequence star & Method \\
\hline Orbital period [h] & \multicolumn{2}{c}{$3.54 \pm 0.07 \mathrm{~h}$} & light curve \\
Inclination & \multicolumn{2}{c}{$\simeq 60^{\circ}$} & radial velocities \\
Distance $[\mathrm{pc}]$ & \multicolumn{2}{c}{$25-40 \mathrm{pc}$} & spectrophotometry \\
$K\left[\mathrm{~km} \mathrm{~s}^{-1}\right]$ & $80 \pm 6$ & $220 \pm 9$ & radial velocities \\
$T_{0}[\mathrm{~d}]$ & $0.566 \pm 0.002$ & $0.052 \pm 0.001$ & radial velocities \\
$T_{0}$ [HJD 2 453 339+] & $0.463 \pm 0.002$ & $0.538 \pm 0.001$ & radial velocities \\
$\gamma\left[\mathrm{km} \mathrm{s}^{-1}\right]$ & $95.0 \pm 5.2$ & $70.0 \pm 6.7$ & radial velocities \\
$T_{\text {eff }} /$ spect. type & $7500 \pm 500 \mathrm{~K}$ & $\mathrm{M} 5 \pm 0.5$ & spectrophotometry \\
Mass $\left[M_{\odot}\right]$ & $0.52 \pm 0.12$ & $0.19 \pm 0.05$ & radial velocities \\
Radius [cm] & $(9 \pm 1) \times 10^{8}$ & $(8 \pm 1.3) \times 10^{9}$ & spectrophotometry \\
\hline
\end{tabular}

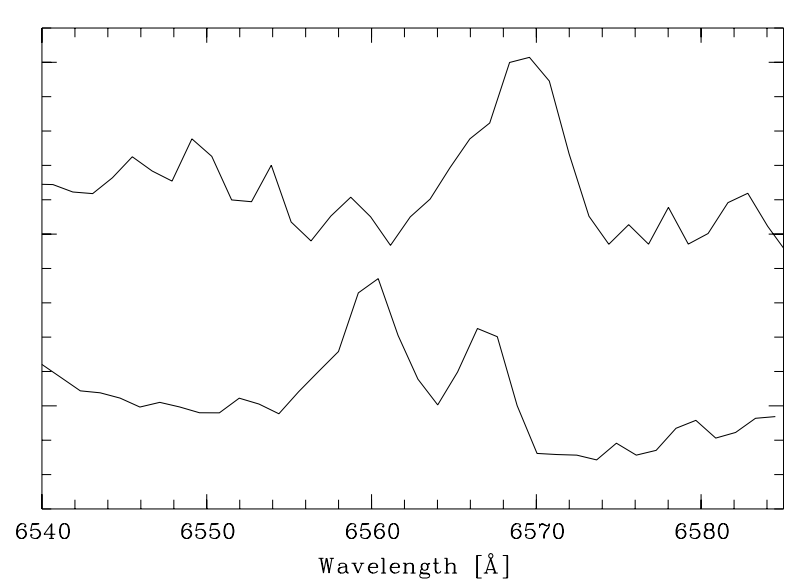

Fig. 7. The $\mathrm{H} \alpha$ line profile at the time of maximum separation of the two emission components (bottom), and half an orbital cycle later (top).

spectral resolution of our data, leaving only their position and amplitude free. For a number of spectra the fit failed to disentangle the two line profiles, especially during those orbital phases when both components cross, and for the spectra from the last third of our run, which suffered from degrading conditions, and where the weaker $\mathrm{H} \alpha$ component was ill-defined. In the cases where one component was still clearly discernable, we fitted the combined profile with two Gaussians as described above, but fixed the position of the weaker component to the velocity predicted by a sine-fit to the radial velocities from the spectra where both components could unambiguously be disentangled. For four spectra, no meaningful result could be extracted from the line profiles. The radial velocity curves derived in this way are shown in Fig. 9.

The two radial velocity variations appear sinusoidal in shape, and are offset by half a cycle. We fitted both sets of radial velocities with sine curves of the form $K \sin \left[2 \pi\left(t-T_{0}\right) / P\right]+\gamma$ with $K$ the radial velocity amplitude, $t$ the time of the observation, $T_{0}$ an offset to account for the arbitrary phase during the observations, $P=0.1475 \mathrm{~d}$ the orbital period determined from the photometry, and a constant velocity offset $\gamma$. The fit parameters are reported in Table 3.

In Fig. 9, we include radial velocities that were derived by fitting a single Gaussian to the absorption edge of the TiO molecular band near $\lambda 7040 \AA$. A sine fit yields the following parameters for this component: $\gamma=89 \pm 5 \mathrm{~km} \mathrm{~s}^{-1}, K=208 \pm 7 \mathrm{~km} \mathrm{~s}^{-1}$, $T_{0}(\mathrm{HJD})=2453339.534 \pm 0.001$, which both in amplitude and phase agrees well with the high-amplitude $\mathrm{H} \alpha$ component, which is also the stronger of the two components.

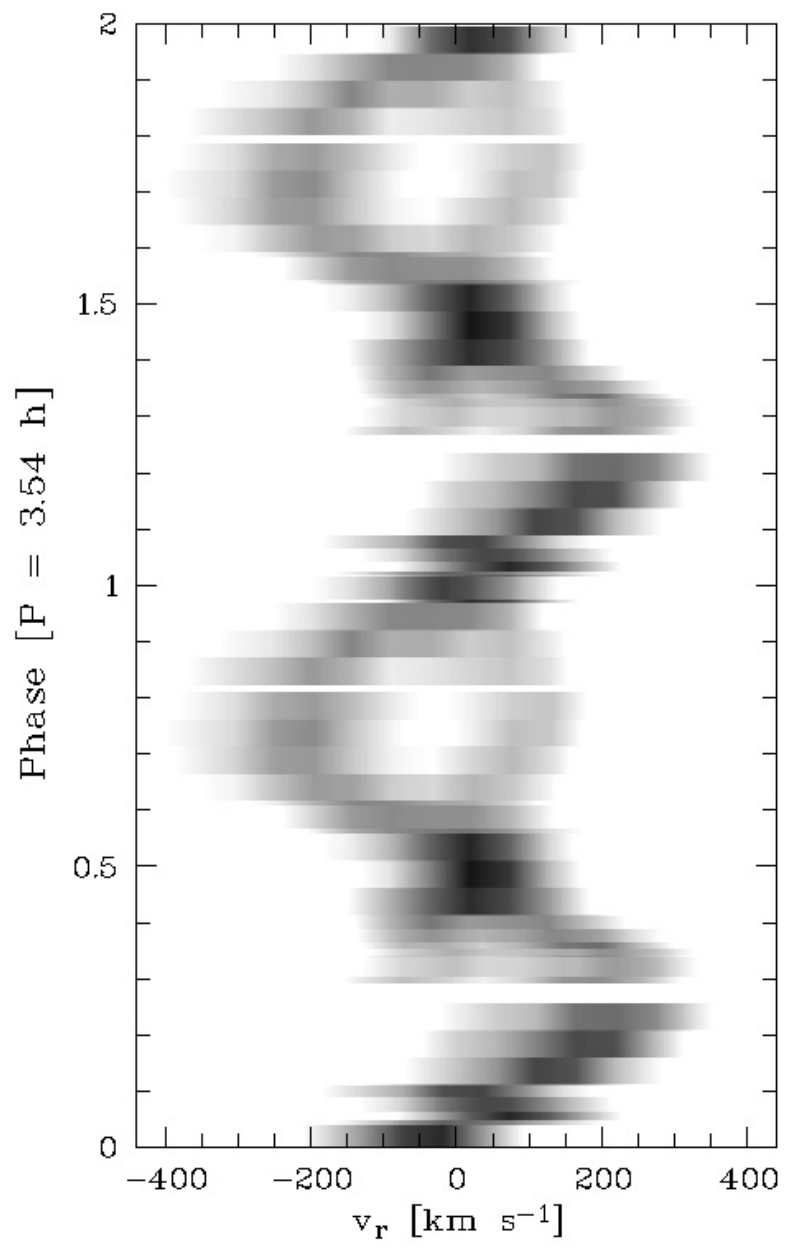

Fig. 8. Trailed spectrogram of the 2004-11-29 data, folded on $P=$ $3.54 \mathrm{~h}$. Radial velocities refer to the rest wavelength of $\mathrm{H} \alpha$. The data are repeated for a second cycle.

We therefore ascribe the two lines moving in anti-phase to emission from the companion star and the white dwarf. Coronal activity in late-type stars is quite common, especially if forced to rapid rotation by being tidally locked in a short-period binary. However, emission from the white dwarf is rather unusual, and may indicate a corona related to low-level accretion from the M-star wind (see O'Donoghue et al. 2003, for a similar phenomenon in the pre-CV EC 13471-1258). Taking such an origin of the two emission lines at face value, their radial velocity variations trace the motion of both stars, and the ratio of their amplitudes equals the mass ratio of the two stars, 


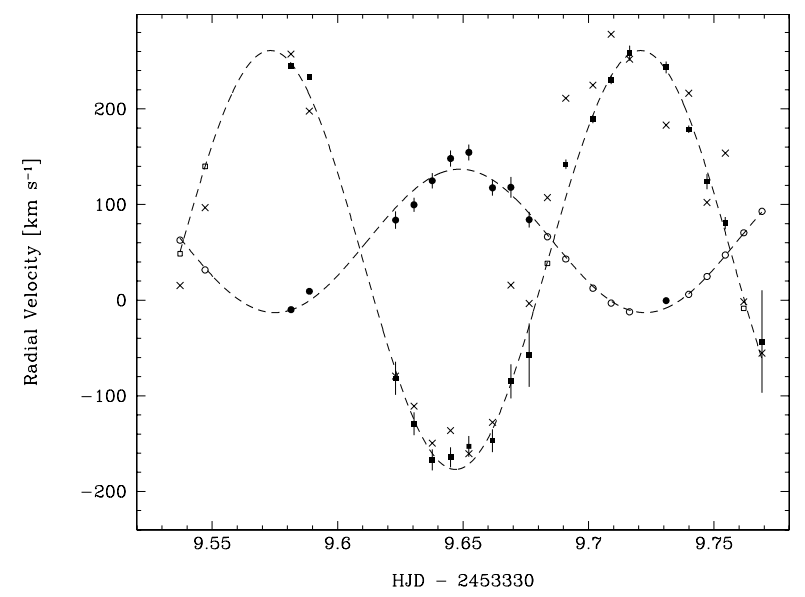

Fig. 9. Radial velocities for both $\mathrm{H} \alpha$ components. For components marked with filled symbols, the velocities were obtained by simultaneous Gaussian fits to both $\mathrm{H} \alpha$ emission line components. In some cases the weaker component was ill-defined, and the two-Gaussian fit was carried out with the position of the weaker component fixed to the wavelength predicted by a sine fit to the velocities obtained from the spectra where a two-Gaussian fit was possible. Those points with fixed velocities are marked by open symbols. Four spectra were too poor to obtain any significant fit, and are marked by open symbols for both components. Additional radial velocities extracted from the $\mathrm{TiO}$ absorption edge at $7040 \AA$ are marked by $\times$. These data were not included in the fit, but are overplotted for comparison.

$K_{\mathrm{wd}} / K_{\mathrm{sec}}=M_{\mathrm{sec}} / M_{\mathrm{wd}}=0.36 \pm 0.03$. We note no substantial change in the equivalent width of the $\mathrm{H} \alpha$ emission line from the companion star, which implies that the motion of the centre of light is equivalent to the motion of the centre of mass. Comparing the velocity offsets obtained from the two fits, it becomes apparent that $\gamma_{\mathrm{wd}}$ exceeds $\gamma_{\mathrm{sec}}$ by $25 \mathrm{~km} \mathrm{~s}^{-1}$. Assuming that $\gamma_{\mathrm{sec}}$ reflects the radial component of systemic velocity, we interpret $\gamma_{\mathrm{wd}}-\gamma_{\mathrm{sec}}$ as the gravitational redshift of the $\mathrm{H} \alpha$ emission originating on the white dwarf. General relativity predicts a redshift of $v_{\mathrm{gr}}=0.635\left(M / M_{\odot}\right) /\left(R / R_{\odot}\right)$, and assuming again a Hamada \& Salpeter (1961) mass-radius relationship, $v_{\mathrm{gr}}=25 \pm 9 \mathrm{~km} \mathrm{~s}^{-1}$ corresponds to $M_{\mathrm{wd}}=0.52 \pm 0.12 M_{\odot}$. A caveat to this mass measurement is that if the $\mathrm{H} \alpha$ emission arises from a point somewhat above the white dwarf photosphere (O'Donoghue et al. 2003, find that the second component of the $\mathrm{H} \alpha$ emission in EC 13471-1258 has its origin somewhere between the white dwarf and $\mathrm{L}_{1}$ ), its mass would be underestimated. The mass of the secondary star implied by the mass ratio above is $M_{\mathrm{sec}}=0.19 \pm 0.05 M_{\odot}$. If the companion star follows the spectral type-mass-radius relation of typical low-mass stars (Ségransan et al. 2003; Delfosse et al. 2000) the masses of both stars are likely to be on the lower side of the error ranges. Given the fact that the mass ratio and both radial velocity amplitudes are known, we can estimate the binary inclination as $\simeq 60^{\circ}$.

\section{Discussion}

In comparison with other known pre-CVs, LTT 560 represents a special case in that it shows spectroscopic evidence for low-level accretion, and apparently irregular long-term variability in its light curve. Accretion from the wind of the companion star has been observed in other pre-CVs in the form of metal enrichment of the white dwarf photosphere or additional emission components (e.g., Sion et al. 1998; O'Brien et al. 2001; O'Donoghue et al. 2003; Maxted et al. 2007). Schmidt et al. (2005, 2007) also ascribe the mass transfer mechansim in low accretion rate polars (LARPS, Schwope et al. 2002) to wind from the secondary star. Concerning the light curve, flaring activity from the secondary star appears as the most likely explanation for the enhanced photometric maximum in the September data, since the $\mathrm{H} \alpha$ component from the secondary star indicates the presence of chromospheric activity, and the duration of the "flare" feature with $\sim 0.15$ orbits $\approx 30 \mathrm{~min}$ is within the usual time range of such flares (e.g., Garcia Alvarez 2000). Still, since the orbital phase in question is only covered once in the September data, it remains unclear whether this feature is not a periodic event, representing an additional light source in the system, perhaps due to enhanced accretion at that time.

A weak X-ray source at a count rate of $0.008 \pm 0.002 \mathrm{cts} / \mathrm{s}$ was serendipitously detected in a pointed ROSAT PSPC observation that coincides within the positional error with LTT 560 (White et al. 2000). Adopting a distance of $33 \mathrm{pc}$ and the countrate-toflux conversion factor of Fleming et al. (1993), we find an X-ray luminosity of $\simeq 6 \times 10^{27} \mathrm{erg} / \mathrm{s}$, which is consistent with coronal activity on the M-dwarf.

A point worth further discussion is the disagreement between the spectrophotometric radius of the secondary star (Sect. 3.1) and that implied by the observed amplitude of the ellipsoidal modulation (Sect.3.2). A well-known problem in the understanding of low mass stars is that the radii of stars in eclipsing binaries with masses of $\simeq 0.4-0.8 M_{\odot}$ are larger by up to $15 \%$ than predicted by stellar models (Ribas 2006 for a review), and stellar activity is generally seen as a possible explanation for this discrepancy (e.g., López-Morales 2007). However, radii of low-mass stars from interferometric measurements also appear to be too large (Berger et al. 2006). In the context of pre-CVs, the companion star is tidally forced into rapid rotation, which is believed to result in increased stellar activity (Pallavicini et al. 1981; Pizzolato et al. 2003). Whether or not stellar activity is the cause of anomalies in the radii, masses, and spectral types of the companion stars in pre-CVs (e.g., O'Brien et al. 2001; Maxted et al. 2004) or CVs (e.g., Naylor et al. 2005) is not yet clear, as no fully detailed treatement of the structure and the observational properties of strongly active low-mass stars is available. In a pioneering paper, Spruit \& Weiss (1986) showed that for stars above the fully convective boundary, both the radius and the temperature of the star will be underestimated if a large fraction of the star is covered by star spots. However, the effects of magnetic activity are thought to extend to lower masses as well (Mullan \& MacDonald 2001), which is confirmed by observations (West et al. 2004). The calculations of Mullan \& MacDonald demonstrated that for low mass M-dwarfs, magnetic activity may result in effective temperatures that are too low for their radii. While a full discussion has to await progress in the theoretical models of active stars, it appears possible that activity caused by rapid rotation induces anomalies in the relationships between mass, radius, and spectral type, and that this is the cause of the apparent mismatch of the spectrophotometric radius of the companion star in LTT560, and its radius implied by the ellipsoidal modulation.

The uncertainty in the stellar properties of the companion star propagates into a relatively wide range of possible periods when LTT 560 will evolve into a semi-detached configuration (i.e. start mass transfer as a cataclysmic variable). For example, using equations (8) and (11) in Schreiber \& Gänsicke (2003), and assuming that gravitational radiation is the main mechanism for angular momentum loss $\left(M_{\mathrm{sec}}<0.3 M_{\odot}\right)$ yields $P_{\mathrm{cd}}=0.165 \mathrm{~d}, P_{\mathrm{sd}}=0.035 \mathrm{~d} \sim 50 \mathrm{~min}$ (which is not a very probable value for a CV), $t_{\mathrm{sd}}=2.9 \mathrm{Gyr}$, for $R_{\mathrm{sec}}=0.115 R_{\odot}$, and $P_{\mathrm{sd}}=0.093 \mathrm{~d} \sim 113 \mathrm{~min}, t_{\mathrm{sd}}=2.0 \mathrm{Gyr}$, for $R_{\mathrm{sec}}=0.22 R_{\odot}$, with 
$P_{\text {cd }}$ being the orbital period at the end of the CE phase, $P_{\text {sd }}$ the orbital period at which the system will start mass transfer (i.e., become a $\mathrm{CV}$ ), and $t_{\mathrm{sd}}$ the time the system will need to shrink from the current orbital period to $P_{\text {sd }}$. Additionally, the spectral type derived in Sect. 3.1 suggests $P_{\mathrm{sd}}<2 \mathrm{~h}$, while the mass ratio determined from the radial velocities and the gravitational redshift indicates $P_{\text {sd }}>3 \mathrm{~h}$. An accurate parallax determination of LTT 560 would therefore be extremely useful for a better constraint on the stellar parameters and the future evolution of LTT 560.

The white dwarf cooling age of LTT 560, assuming $M_{\mathrm{wd}} \simeq$ $0.5 M_{\odot}$, is $\simeq 10^{9} \mathrm{yr}($ Wood 1995$)$. The relatively low mass suggests that it may contain a helium core, which is a possible outcome of the common envelope evolution (Willems \& Kolb 2004 and references therein). Better data are needed to improve the measurement of the white dwarf mass.

LTT 560 and RR Cae (Bragaglia et al. 1995; Bruch \& Diaz 1998; Bruch 1999) represent the oldest among the $\sim 40$ known post-common envelope binaries (Schreiber \& Gänsicke 2003; Morales-Rueda et al. 2005; Shimansky et al. 2006). Both systems are nearby $(\simeq 33 \mathrm{pc}$ and $\simeq 12 \mathrm{pc}$, respectively) which suggests that they represent a fairly numerous population, as predicted to exist by Schreiber \& Gänsicke (2003). A principle difficulty in finding old pre-CVs is their inconspicuousness compared to CVs. Both stellar components in old pre-CVs are intrinsically faint, and hence only nearby systems will be found in shallow surveys. Pre-CVs do not undergo outburst, so will not be picked up by amatuer astronomers of sky patrols. They have at best some X-ray emission due to coronal activity of the main sequence companion, indistinguishable from single M-dwarfs. Old pre-CVs are not particularly blue, so escaped discovery in the classic "blue" extragalactic surveys such as the Palomar Green Survey. The Sloan Digital Sky Survey (SDSS), being deeper and sampling a much larger colour space than any previous survey, is now rapidly changing the scene, identifying already $\gtrsim 1000$ white dwarf main sequence binaries (Silvestri et al. 2006; Eisenstein et al. 2006; Southworth et al. 2007), with more to come from SEGUE (Schreiber et al. 2007). However, follow-up work identifying post-CE/pre-CV binaries among those objects has just started (e.g. Rebassa-Mansergas et al. 2007), and will be limited (by the design of SDSS) predominantly to relatively faint systems. While SDSS will substantially improve our knowledge on pre-CVs in terms of better statistics, identifying nearby bright pre-CVs such as LTT 560 white dwarf main sequence binaries remains important as those systems are best-suited for detailed parameter studies.

Acknowledgements. This research has greatly benefited from discussions with Tom Marsh, Matthias Schreiber, Raymundo Baptista, and Stella Kafka. We also thank the anonymous referee for helpful suggestions.

C.T. and R.M. acknowledge financial support by FONDECYT grant 1051078 . BTG was supported by a PPARC Advanced Fellowship. A.A. thanks the Royal Thai Government for a studentship. This work has made intensive use of the SIMBAD database, operated at CDS, Strasbourg, France, and of NASA's Astrophysics Data System Bibliographic Services. IRAF is distributed by the National Optical Astronomy Observatories.

\section{References}

Beer, M. E., Dray, L. M., King, A. R., \& Wynn, G. A. 2007, MNRAS, 375, 1000 Berger, D. H., Gies, D. R., McAlister, H. A., et al. 2006, ApJ, 644, 475 Bessell, M. S. 1990, PASP, 102, 1181 Beuermann, K. 2006, A\&A, 460, 783
Beuermann, K., \& Weichhold, M. 1999, in Annapolis Workshop on Magnetic Cataclysmic Variables, ed. C. Hellier, \& K. Mukai, ASP Conf. Ser., 157, 283 Bochanski, J. J., West, A. A., Hawley, S. L., \& Covey, K. R. 2007, AJ, 133, 531 Bragaglia, A., Renzini, A., \& Bergeron, P. 1995, ApJ, 443, 735

Bruch, A. 1999, AJ, 117, 3031

Bruch, A., \& Diaz, M. P. 1998, AJ, 116, 908

de Kool, M. 1992, A\&A, 261, 188

Delfosse, X., Forveille, T., Ségransan, D., et al. 2000, A\&A, 364, 217

Downes, R., Webbink, R. F., \& Shara, M. M. 1997, PASP, 109, 345

Eggen, O. J. 1968, ApJ, 153, 195

Eisenstein, D. J., Liebert, J., Harris, H. C., et al. 2006, ApJS, 167, 40

Fleming, T. A., Giampapa, M. S., Schmitt, J. H. M. M., \& Bookbinder, J. A. 1993, ApJ, 410, 387

Garcia Alvarez, D. 2000, Ir. Astron. J., 27, 117

Hamada, T., \& Salpeter, E. E. 1961, ApJ, 134, 683

Hoard, D. W., \& Wachter, S. 1998, PASP, 110, 906

Horne, K. 1986, PASP, 98, 609

Howell, S. B. 1992, in Astronomical CCD Observing and Reduction Techniques, ASP Conf. Ser., 23, ed. S. B. Howell

Koester, D., Napiwotzki, R., Voss, B., Homeier, D., \& Reimers, D. 2005, A\&A, 439, 317

López-Morales, M. 2007, ApJ, 660, 732

Luyten, W. J. 1957, A catalogue of 9867 stars in the Southern Hemisphere with proper motions exceeding 0. '2 annually (Minneapolis: Lund Press)

Maxted, P. F. L., Marsh, T. R., Morales-Rueda, L., et al. 2004, MNRAS, 355 , 1143

Maxted, P. F. L., O’Donoghue, D., Morales-Rueda, L., Napiwotzki, R., \& Smalley, B. 2007, MNRAS, 376, 919

Meyer, F., \& Meyer-Hofmeister, E. 1979, A\&A, 78, 167

Morales-Rueda, L., Marsh, T. R., Maxted, P. F. L., et al. 2005, MNRAS, 359, 648

Mullan, D. J., \& MacDonald, J. 2001, ApJ, 559, 353

Naylor, T., Allan, A., \& Long, K. S. 2005, MNRAS, 361, 1091

O'Brien, M. S., Bond, H. E., \& Sion, E. M. 2001, ApJ, 563, 971

O’Donoghue, D., Koen, C., Kilkenny, D., et al. 2003, MNRAS, 345, 506

Paczyński, B. 1976, in Structure and Evolution of Close Binary Systems, ed. P. Eggleton, S. Mitton, \& J. Whelan, IAU Symp., 73, 75

Pallavicini, R., Golub, L., Rosner, R., et al. 1981, ApJ, 248, 279

Pizzolato, N., Maggio, A., Micela, G., Sciortino, S., \& Ventura, P. 2003, A\&A, 397,147

Rebassa-Mansergas, A., Gänsicke, B., Rodríguez-Gil, P., Schreiber, M., \& Koester, D. 2007, MNRAS, submitted

Ribas, I. 2006, Ap\&SS, 304, 89

Ritter, H., \& Kolb, U. 2003, A\&A, 404, 301

Schmidt, G. D., Szkody, P., Vanlandingham, K. M., et al. 2005, ApJ, 630, 1037

Schmidt, G. D., Szkody, P., Henden, A., et al. 2007, ApJ, 654, 521

Schreiber, M., Nebot Gomez-Moran, A., \& Schwope, A. 2007, in 15th European

Workshop on White Dwarfs, ed. R. Napiwotzki, \& R. Burleigh, ASP Conf.

Ser., in press, [arXiv: astroph/0611461]

Schreiber, M. R., \& Gänsicke, B. T. 2003, A\&A, 406, 305

Schwarzenberg-Czerny, A. 1989, MNRAS, 241, 153

Schwope, A. D., Brunner, H., Hambaryan, V., \& Schwarz, R. 2002, in The Physics of Cataclysmic Variables and Related Objects, ed. B. T. Gänsicke, K. Beuermann, \& K. Reinsch, ASP Conf. Ser., 261, 102

Ségransan, D., Kervella, P., Forveille, T., \& Queloz, D. 2003, A\&A, 397, L5

Shimansky, V., Sakhibullin, N. A., Bikmaev, I., et al. 2006, A\&A, 456, 1069

Silvestri, N. M., Hawley, S. L., West, A. A., et al. 2006, AJ, 131, 1674

Sion, E. M., Schaefer, K. G., Bond, H. E., Saffer, R. A., \& Cheng, F. H. 1998, ApJ, 496, L29

Southworth, J., Gänsicke, B., \& Schreiber, M. 2007, MNRAS, submitted

Spruit, H. C., \& Weiss, A. 1986, A\&A, 166, 167

Stetson, P. B. 1992, in Astronomical Data Analysis Software and Systems I, ed. D. M. Worrall, C. Biemesderfer, \& J. Barnes, ASP Conf. Ser., 25, 297

Tappert, C., Gänsicke, B. T., Schmidtobreick, L., Mennickent, R. E., \& Navarrete, F. P. 2007, A\&A, in press, [arXiv:0707.0501]

Vogt, N. 1989 (Chichester: Wiley, ed. M. F Bode, A. Evans, 225

Warner, B. 1995, Cataclysmic variable stars, Cambridge Astrophysics Series (Cambridge, New York: Cambridge University Press)

West, A. A., Hawley, S. L., Walkowicz, L. M., et al. 2004, AJ, 128, 426

White, N. E., Giommi, P., \& Angelini, L. 2000, VizieR Online Data Catalog, 9031, 0

Willems, B., \& Kolb, U. 2004, A\&A, 419, 1057

Wood, M. A. 1995, in White Dwarfs, ed. D. Koester, \& K. Werner (Heidelberg: Springer), LNP, 443, 41 